\title{
Immunologic research at Johns Hopkins University: introduction and overview
}

\author{
M. Sue Leffell
}

Published online: 23 January 2010

(C) Springer Science+Business Media, LLC 2010

There is a rich heritage of immunologic research at the Johns Hopkins University that has developed into many diverse programs. The lead article for this issue is by Noel R. Rose, who provides an engaging history of immunologic research at Johns Hopkins which began with the creation of the first department devoted to immunology in this country. Dr. Rose details the growth and expansion of immunologic research initiated by William Henry Welch that fostered not only the current programs at Johns Hopkins, but also the research at other institutions. At Johns Hopkins, the initial research programs have expanded into multiple centers exploring basic immunology as well as innovative, translational research applicable to clinical practice.

The articles in this issue provide a representative, but by no means inclusive, perspective of current Johns Hopkins immunologic research programs. There are several reviews that examine basic immunologic mechanisms, ranging from the molecular mechanisms controlling somatic hypermutation in immunoglobulin variable and switch regions to the genetic and biochemical regulation of lymphocyte development and differentiation. Two articles provide insights into how the conformation of different peptides impact antigen presentation by major histocompatibility complex (MHC) class II molecules, and how MHC class I molecules can be used to probe the patchiness and heterogeneity of cell surfaces. Other innovative work illustrates how MHC-immunoglobulin complexes are utilized as part of artificial antigen-presenting cells that provide platforms for investigating both in vitro and in vivo immune responses.

The next series of reviews highlight research programs investigating immunologic mechanisms contributing to autoimmunity, allergy and inflammation, infectious diseases, and immunoparasitology. These topics include signaling pathways in allergic inflammation;

\footnotetext{
M. S. Leffell ( $\bowtie)$

Department of Medicine, Johns Hopkins University School of Medicine, Baltimore, USA e-mail: msl@jhmi.edu
}

M. S. Leffell

Bloomberg School of Public Health, Baltimore, USA

M. S. Leffell

Immunogenetics Laboratory, 2041 East Monument Street, Baltimore, MD 21205, USA 
the role of IgE specific activity in allergic reactions; environmental triggers in autoimmunity; cytokine regulation in inflammation and ocular disease; immune-mediated recovery from viral encephalitis; and the immune evasive mechanisms contributing to persistent Hepatitis $\mathrm{C}$ viral infection and chronic visceral leishmaniasis.

Translational research is the focus of many Johns Hopkins laboratories and this issue highlights three areas, vaccine development, immunotherapy for autoimmunity, and immune modulation for solid organ and hematopoietic stem cell transplantation. Vaccine development for prevention and/or treatment of malignancies is an exciting area of immunologic research, and novel approaches toward the immunotherapy of human papillomavirus associated cervical cancer are presented. Both hematopoietic stem cell and solid organ transplantation have been long-standing programs at Johns Hopkins and have fostered basic, as well as more translational research. Articles representative of more basic research in these areas discuss reconstitution of self-tolerance, mechanisms of antibody/ complement-mediated rejection, and immunologic factors in ischemia and reperfusion injury. Researchers in hematology and oncology have developed innovative protocols using high-dose cyclophosphamide therapy to "re-program the immune system." These protocols have been successfully applied to the treatment of autoimmune disorders as well as facilitating graft-host tolerance following hematopoietic stem cell transplantation. Humoral sensitization to HLA antigens has been a major barrier to transplantation, but the development of desensitization protocols and programs for paired living donor exchange is facilitating successful renal transplantation of highly sensitized patients. Members of the Comprehensive Transplant Center at Johns Hopkins have been leaders in desensitization and paired kidney exchange and share their experience over the last decade. The authors of the last two articles address the lessons learned from a clinical perspective as well as those from the immunogenetic and transplant immunology view.

On behalf of the other guest editors, Andrea A. Zachary and Noel R. Rose, I extend thanks to the Editor and Publishers of Immunologic Research for the opportunity to highlight the excellent and innovative work of our colleagues. 